\title{
Mycological Profile and Its Associated Factors Among Patients Suspected of Dermatophytosis at Bisidimo Hospital, Eastern Ethiopia
}

\author{
Mina Ali Dawa' \\ Tewodros Tesfa (iD ${ }^{2}$ \\ Fitsum Weldegebreal (iD) ${ }^{2}$ \\ 'Department of Veterinary Medicine, \\ College of Veterinary Medicine, Haramaya \\ University, Haramaya, Ethiopia; \\ ${ }^{2}$ Department of Medical Laboratory \\ Sciences, College of Health and Medical \\ Science, Haramaya University, Harar, \\ Ethiopia
}

Background: Dermatophytosis is a common problem across the globe that is caused by a group of closely related fungi known as the dermatophytes that can invade keratinized tissues. It is a usual refractory infection and deleteriously affects the quality of life of humans. Despite the common presence of dermatophytes on the human host, research evidence in this area is limited. Objective: Aimed to assess the mycological profile and its associated factors among patients suspected of dermatophytosis at Bisidimo Hospital, Eastern Ethiopia, from March to October 2021.

Methods: A cross-sectional study was conducted on 289 patients who visited the dermatology department of Bisidimo Hospital. Data were collected by reviewing patients' charts and using a structured questionnaire to assess the associated factors of dermatophytosis. The patients' medical charts were reviewed to assess chronic conditions. Skin, hair, or nail samples were collected and transported to Medical Microbiology Laboratory at Haramaya University Department of Medical Laboratory Sciences. Samples were inoculated into Potato dextrose agar and Sabouraud dextrose agar, and fungal isolates were identified morphologically and microscopically. Data were analyzed using Statistical Package for the Social Sciences version 26. Bivariate and multivariable logistic regression analyses with $95 \%$ confidence intervals were carried out to identify factors associated with dermatophytosis.

Results: The overall magnitude of dermatophytosis infection was $28.4 \%$ (95\%CI: $23.2-$ 32.2). Tinea capitis and tinea corporis were the common clinical presentations. The fungal isolates include Trichophyton species (68 isolates) and Epidermophyton species (17 isolates). Patients with features involving male sex $(\mathrm{AOR}=2.5)$, age group of $1-10$ years $(\mathrm{AOR}=$ $3.5)$, hypertension $(\mathrm{AOR}=2.2)$, a family history of dermatophytosis $(\mathrm{AOR}=2.0$ ), and a history of animal contact $(\mathrm{AOR}=1.5)$ had the increased odds of being infected with the dermatophytosis.

Conclusion: We found more than one in four patients assessed had dermatophytosis infection. It was considerably high in patients with a family history of the infection, animal contacts, hypertension, and below 10 years of age. Therefore, the control program for this disease should specifically target at a high risk populations, which must hinge on the evidence of molecular characterization and antifungal susceptibility patterns of the fungi.

Keywords: dermatophytosis, mycological profile, patients, Bisdimo, Ethiopia

\section{Introduction}

Dermatophytosis is a fungal infection of the skin, nails, and hair in humans and animals caused by a group of closely related pathogens called dermatophytes. ${ }^{1}$
Correspondence: Fitsum Weldegebreal Department of Medical Laboratory Sciences, College of Health and Medical Science, Haramaya University, P.O. Box: 235, Harar, Ethiopia Email fwmlab2000@gmail.com 
Three asexual genera of fungi are the etiological agents (i. e.,Trichophyton, Microsporum, and Epidermophyton). The most common species that cause disease in humans are Trichophyton rubrum, Trichophyton tonsurans, Trichophyton interdigitale, Trichophyton mentagrophytes, Microsporum canis, and Epidermophyton floccosum. ${ }^{2}$

The laboratory diagnosis of dermatophytosis involves direct microscopic examination, culture and molecular techniques. The microscopic examination is routinely practiced as it is a rapid diagnostic method though it lacks specificity and sensitivity, with up-to $15 \%$ falsenegative results. A fungal culture is a specific diagnostic test, but it is slow. Above all, the molecular technique is a highly sensitive and specific test. ${ }^{3,4}$

Dermatophytes have been reported all over the world with variations in their epidemiologic, distributions, incidence, and hosts. Factors responsible for these variations include geographic location and climate (temperature, humidity, wind, etc.), hygiene, overcrowding, culture, health care, immigration, and other factors (i.e., age, sex, season, and socioeconomic factors). ${ }^{3}$ Dermatophytosis can lead to a generalized and atypical presentation that can be confused with other skin disorders in immunocompromised people (e.g., patients with acquired immunodeficiency syndrome, cancer treatment, immunosuppressive drugs, diabetes mellitus and Cushing's disease). ${ }^{5}$ Furthermore, contact with infected animals, sharing of fomites and illiteracy have been associated with dermatophyte infections in developing countries. ${ }^{6}$

The prevalence of dermatophytosis is high in developing nations located in the tropic with wet humid climates with an estimated overall incidence of tinea to be 78 million in sub-Saharan Africa. ${ }^{7}$ Though dermatophytosis occurs in both immunocompromised patients and healthy individuals. ${ }^{8}$ There is an increased occurrence of these infections in recent years mainly due to change in lifestyle and climatic conditions, inappropriate excessive use of antibiotics, and immunosuppression. ${ }^{9}$

It is a typical refractory infection that upsets day-to-day activities and social stigmas that leads to deleterious impacts on the quality of life of people. ${ }^{3}$ It is a costly disease in terms of psychological effects, treatments, and loss of working days. ${ }^{10}$

Despite the constant presence, only a few studies have investigated the etiology of cutaneous fungal infections in the developing world including Ethiopia. Therefore, this study was undertaken to assess the mycological profile and its associated factors among patients suspected of dermatophytosis at Bisidimo Hospital, eastern Ethiopia.

\section{Materials and Methods}

\section{Study area and period}

The study was conducted in Bisidimo hospital from March to October 2021. It is found in East Hararghe, Oromia region, Ethiopia. It is located in the Erer valley, about $22 \mathrm{Km}$ to the east of Harar town at the end of a turn-off from the road to Jigjiga. The area is a semi-arid planed at around $1400 \mathrm{~m}$ surrounded by a chain of rocky hills. Bisidimo Hospital was previously a reference center for leprosy and dermatological disease. Currently, the hospital has surgical, medical, gynecology, pediatrics, dermatology, ophthalmology, and dental departments. ${ }^{11}$

\section{Study design and populations}

A hospital-based cross-sectional study was conducted. All patients suspected of dermatophytosis fulfilled the inclusion criteria, and patients who had complete information on their medical chart during the study period were included. Patients already on treatment for fungal infection and those with an open wound or subcutaneous mycosis and those patients who had incomplete information on their medical chart were excluded.

\section{Sample size determination and sampling procedure}

The sample size was determined by single population proportion formula with the assumption of $95 \%$ confidence level, $5 \%$ margin of error, and a prevalence rate of $23.4 \%$ from a previous study conducted in the Harari region. ${ }^{12}$ The final sample size with a $5 \%$ non-response rate adjusted was thus 289 , and a purposive sampling technique was employed for selecting the patients.

\section{Data collection methods}

The data were collected by two laboratory professionals who were supervised by a senior BSc laboratory professional. We collected the data using pretested structured questionnaire and a checklist prepared by reviewing different literature. ${ }^{13,14}$ The questionnaire was prepared in English and translated into the local language (Afan Oromo) and back-translated to English to confirm consistency. The data were collected by face-toface interviews after written consent was obtained from study participants/guardians alongside the collection of sample. And, medical charts were reviewed to assess clinical features 
of the patients that include diabetes, hypertension, and HIV/ AIDS status.

\section{Specimen collection and transportation}

The specimen collection was carried out using the procedure described by Ardakani et al. ${ }^{15}$ Scrapings from the skin consisting of epidermal scales and from the infected hairs were taken using a sterile scalpel blade on a piece of black paper and toothbrush, respectively, by laboratory professionals after cleaning the affected sites with $70 \mathrm{v} / \mathrm{v}$ alcohol. Each specimen was labeled and transported by paper packages within 24 hours for microscopic examination and culture.

\section{Direct microscopic examination}

Collected specimens were first softened and cleared with a strong alkali of $(20 \% \mathrm{w} / \mathrm{v})$ potassium hydroxide $(\mathrm{KOH})$. Next, a drop of $\mathrm{KOH}$ solution was placed on a microscopic slide. Then, part of the specimens $(5 \mathrm{~mm}$ of hairs, and $2 \mathrm{~mm}$ of skin scales and crusts) were added to the drop of $\mathrm{KOH}$ and covered with a cover glass. Following this, the slide was placed in a Petri dish, or another container with a lid, together with a damp piece of filter paper or cotton wool to prevent the preparation from drying out, as soon as the specimen was cleared. Finally, it was examined microscopically using the 10x and 40x objectives. And, the slides were scanned for the presence of branching septate hyphae with angular or spherical arthroconidia (arthrospores), usually in chains. ${ }^{16}$

\section{Fungal culture}

Culture procedure started with plating clinical samples onto a properly selected general media Potato dextrose agar (HiMeadia, India) with added antibiotics (chloramphenicol $50 \mathrm{mg} / \mathrm{L}$ ) to inhibit overgrowth by bacterial contaminants and a selective media Sabouraud dextrose agar (SDA) (HiMedia, India) base supplemented with chloramphenicol (which inhibits bacteria) and cycloheximide (500 mg/L) (to inhibit saprophytic fungi). Cultures were incubated aerobically at room temperature $\left(25^{\circ} \mathrm{C}\right)$ for up to 4 weeks for the better and more rapid growth of nearly all pathogenic fungi. We used colony texture, growth rate, topography, and pigmentation of the front and the reverse side of the culture for the macroscopic identification. The microscopic identification of mold isolates was performed by placing pieces of a colony from SDA and/ or PDA to clean microscopic slides and stain them with lactophenol cotton blue. After placing a coverslip, each of the slide preparation was observed microscopically. ${ }^{16}$

\section{Data quality control}

We gave training to data collectors and supervisors for two days. The data collection instrument was pretested in $5 \%$ of the sample size in the hospital but we did not include these data in the actual study. Next, necessary modifications were made based on the pretest feedbacks to make the questionnaire clear and understandable. All culture media were prepared following the manufacturer's instructions, and sterility was checked by incubating $3-5 \%$ of the batch at $37^{\circ} \mathrm{C}$ overnight and observing for the growth of bacteria and saprophytic fungi. Culture media batch which showed any contamination was discarded and replaced by a new sterile medium. We performed tests based on the recommended standard operating procedure (SOP). The completeness of data was checked on a daily basis.

\section{Methods of data analysis}

Data were double entered into EPI-Data version 3.1 and transferred to Statistical Package for Social Science (SPSS) program version 26 (IBM Company) for analysis. Descriptive statistics of different variables were determined and expressed in the form of texts, tables, and graphs using summary measures such as percentages, mean and median.

Bivariate logistic regression was carried out to identify the associated factors with dermatophyte infection. Variables with a $\mathrm{p}$-value $\leq 0.25$ in the bivariable analysis were candidates for the multivariable model building. The multi-collinearity test was carried out to observe the correlation between predictor variables using standard error and independent variables analysis, and a variable with a standard error of $>2$ was rejected. The degree of statistical significance was declared at a p-value $<0.05$ with $95 \%$ confidence intervals.

\section{Ethical considerations}

Ethical clearance was obtained from the Institutional Health Research Ethical Review Committee (IHRERC) of the College of Health and Medical Sciences Haramaya University. The study was conducted in accordance with the Declaration of Helsinki. A support letter was obtained from the College of Health and Medical Sciences to Bisidimo Hospital and by explaining the objectives of the study and its significance, permission was obtained from the hospital. At the individual level after explaining the purpose of the study, informed voluntary and written consent was obtained from all participants/guardians before they were 
Table I Socio-Demographic Characteristics of Dermatophytosis Suspected Patients in Bisidimo Hospital, Eastern Ethiopia, 202I $(n=289)$

\begin{tabular}{|c|c|c|c|}
\hline \multicolumn{2}{|c|}{ Demographic Characteristics } & \multirow{2}{*}{$\begin{array}{l}\text { Frequency } \\
195\end{array}$} & \multirow{2}{*}{$\begin{array}{l}\text { (\%) } \\
67.5\end{array}$} \\
\hline Sex & Male & & \\
\hline & Female & 94 & 32.5 \\
\hline \multirow[t]{4}{*}{ Age } & $1-10$ & 72 & 24.9 \\
\hline & $I I-20$ & 95 & 32.9 \\
\hline & $21-30$ & 67 & 23.2 \\
\hline & $>30$ & 55 & 19.03 \\
\hline \multirow[t]{2}{*}{ Residence } & Rural & $15 \mid$ & 52.2 \\
\hline & Urban & 138 & 47.8 \\
\hline \multirow[t]{5}{*}{ Occupation } & Farmer & 71 & 24.6 \\
\hline & Student & 133 & 46 \\
\hline & House wife & 44 & 15.2 \\
\hline & Others & 25 & 8.6 \\
\hline & Government & 16 & 5.5 \\
\hline \multirow{3}{*}{$\begin{array}{l}\text { Educational } \\
\text { level }\end{array}$} & Illiterate & 55 & 19.03 \\
\hline & Primary school & 54 & 13.8 \\
\hline & $\begin{array}{l}\text { Secondary } \\
\text { school }\end{array}$ & 180 & 62.2 \\
\hline
\end{tabular}

participated in this study. Participants were assured that confidentiality was maintained and the completed questionnaire was kept anonymous.

\section{Results}

\section{Sociodemographic characteristics}

A total of 289 dermatophytosis suspected patients were enrolled. The age of the study subjects ranged from 1 to 52 years with a mean age of $20(\mathrm{SD} \pm 10.3)$ years, and 195 $(67.5 \%)$ were males. Among the study participants, 133 (46\%) of them were students, 151 (52.2\%) were from a rural area, and $180(62.2 \%)$ had attended secondary school (Table- 1).

\section{Clinical characteristics}

Out of 289 study participants, 193 (66.8\%) had a family history of dermatophytosis, of which 104 (35.9\%) did not get treatment for the infection. Again, $82(28.3 \%)$ of the patients shared fomites, and $151(52.2 \%)$ of them had a history of animal contact. Twelve $(4.15 \%)$ of the patients had
Table 2 Clinical Characteristics of Dermatophytosis Suspected Patients in Bisidimo Hospital, Eastern Ethiopia, 202I ( $n=289)$

\begin{tabular}{|l|l|l|l|}
\hline \multicolumn{2}{|l|}{ Characteristics } & Frequency & Percent (\%) \\
\hline Family history & Yes & 193 & 66.8 \\
\cline { 2 - 4 } & No & 96 & 33.2 \\
\hline \multirow{3}{*}{ Treated for dermatophytosis } & Yes & 94 & 32.5 \\
\cline { 2 - 4 } & No & 104 & 35.9 \\
\hline \multirow{3}{*}{ Share fomites } & Yes & 82 & 28.3 \\
\cline { 2 - 4 } & No & 27 & 9.34 \\
\hline Animal contact & Yes & 151 & 52.2 \\
\cline { 2 - 4 } & No & 138 & 47.8 \\
\hline Diabetic & Yes & 22 & 7.6 \\
\cline { 2 - 4 } & No & 267 & 92.4 \\
\hline Hypertension & Yes & 12 & 4.15 \\
\cline { 2 - 4 } & No & 277 & 95.8 \\
\hline HIV/AIDS & Yes & 58 & 20.06 \\
\cline { 2 - 4 } & No & 231 & 79.93 \\
\hline
\end{tabular}

hypertension, $22(7.6 \%)$ were diabetic patients, and 58 $(20.06 \%)$ were HIV/AIDS positive patients (Table-2).

\section{Magnitude of dermatophytosis}

The overall prevalence of dermatophytosis was $28.4 \%$ (95\% CI (23.2-32.2)). Out of 289 suspected cases, about $49.1 \%$ were diagnosed clinically. Fungi were detected in $33.9 \%$ using potassium hydroxide wet mount, and $28.4 \%$ using culture technique. From the culture fungi isolates $79.26 \%$ of them were Trichophyton. Prevalence of dermatophytosis based on culture results showed that the highest prevalence was in males $(79.26 \%)$, age groups between 1 and 10 years $(45.1 \%)$, students $(60.9 \%)$, urban residents $(51.2 \%)$, and individuals who have attended secondary schools $(71.9 \%)$. Out of 142 cases that were diagnosed clinically, $73.2 \%$ were males. Tinea capitis $(41.3 \%)$ and Tinea corporis $(44.7 \%)$ were the commonest clinical presentation among males and females, respectively (Table 3).

\section{Associated Factors}

Sex, age, residence, occupation, family history, sharing of fomites, animal contact, and hypertension have a P-value 


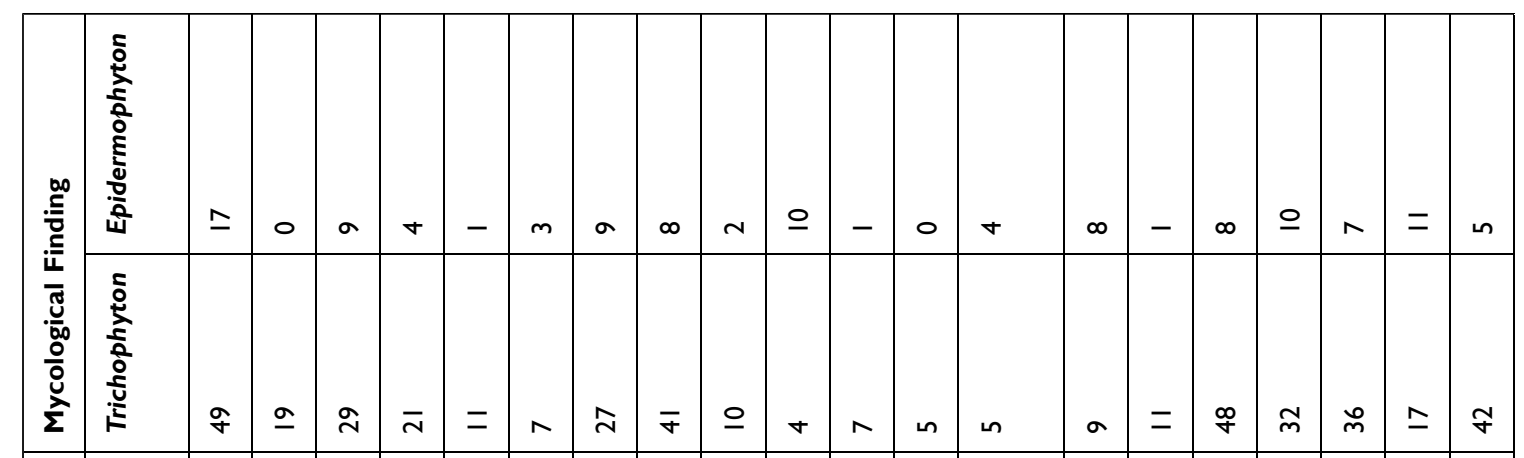

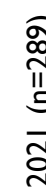

$\frac{\frac{\pi}{0}}{\frac{0}{\bar{t}}}$

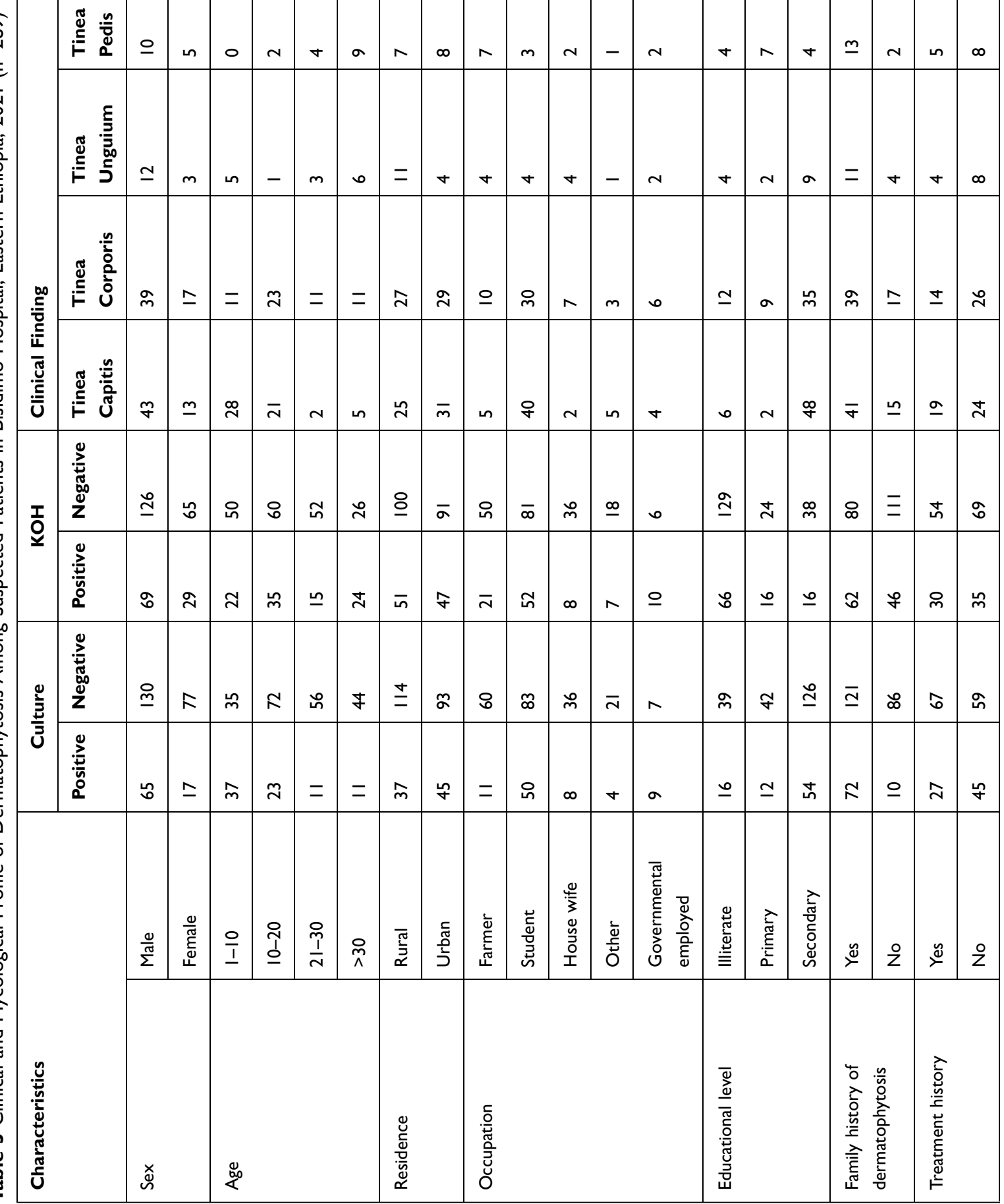




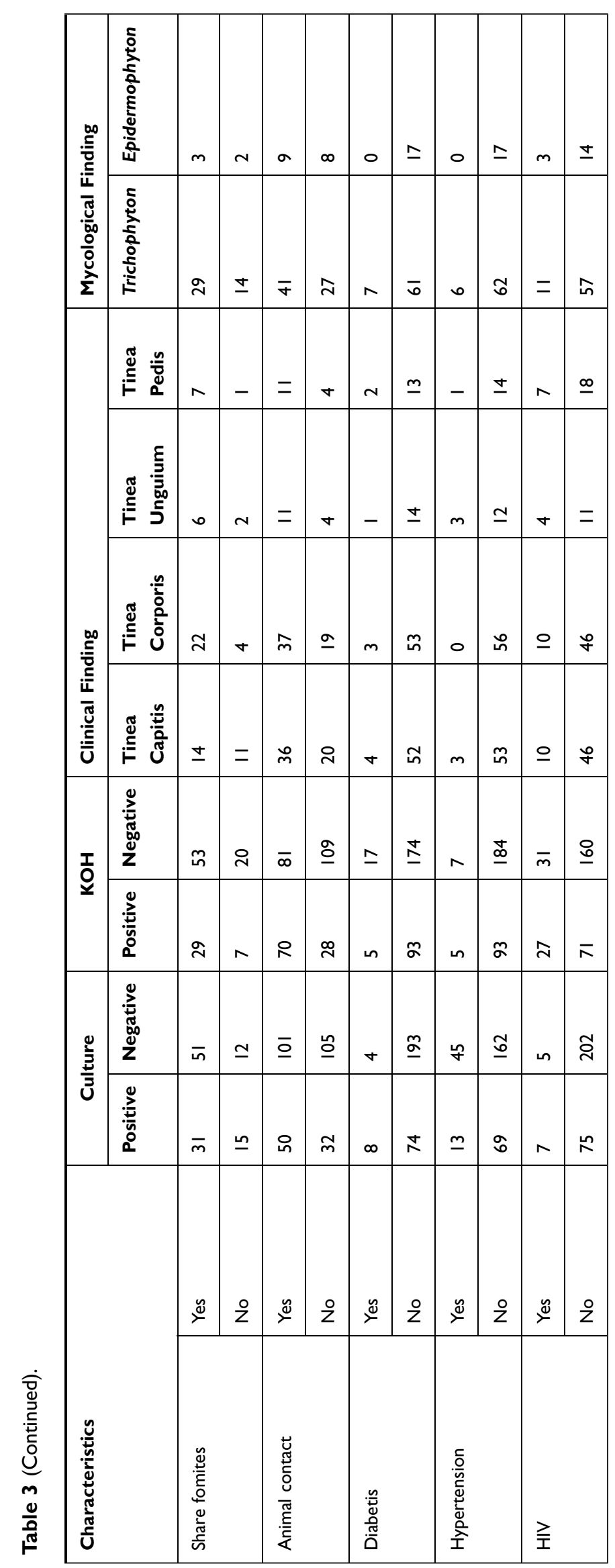


less than 0.25 in bivariable regression analysis, and they were considered as candidates for the multivariable logistic regression analysis. In multivariable analysis, features of patients inlcuding sex, age, family history of animal contact, and hypertension remained statistically significant at P-value less than 0.05 .

Males were 2.5-times $(\mathrm{AOR}=2.5 ; 95 \% \mathrm{CI}: 1.96,4.80)$ more likely to be infected with dermatophytosis compared to females. Patients 1 to 10 years of age were 3.5-times $(\mathrm{AOR}=3.5 ; 95 \% \mathrm{CI}: 2.07,5.57)$ more likely to be infected compared to those aged greater than 30 years. Patients who had a previous family history of dermatophytosis had 2 times $(\mathrm{AOR}=2.0 ; 95 \% \mathrm{CI}: 1.03,5.14)$ more likely odds of developing dermatophytosis. Those patients who had a history of contact with animals were 1.5 times $(\mathrm{AOR}=1.5 ; 95 \% \mathrm{CI}: 1.07,4.57)$ more likely to be infected than their counterparts. Hypertensive patients were 2.2 times $(\mathrm{AOR}=2.2 ; 95 \% \mathrm{CI}: 1.12-7.08)$ more likely to be infected than their counterparts (Table 4).

\section{Discussion}

This clinical and mycological study was done on 289 dermatophytosis suspected patients in the dermatology department at Bisidimo Hospital. In the present study, $49.13 \%, 33.9 \%$, and $28.4 \%$ were diagnosed for dermatophytosis clinically, $\mathrm{KOH}$ direct microscopy, and culture, respectively. This study highlights the importance of both $\mathrm{KOH}$ preparation and culture in the diagnosis of dermatophytosis. However, culture gives a definitive diagnosis with the type of fungal species given that $\mathrm{KOH}$ identifies all fungus non-specifically. ${ }^{17}$

In the current study, the magnitude of dermatophytosis infection was $28.4 \%$. This finding is consistent with previous reports from Harar, Ethiopia (23.4\%), ${ }^{12}$ and Iran $(29.4 \%) .{ }^{18}$ However, higher rates of this infection were reported from the studies conducted in Addis Ababa, Ethiopia $(42.6 \%)^{13}$ and $(85.2 \%) .{ }^{19}$ This variation between the studies might be explained by differences in sample size, variation in the study population, the difference in methods of diagnosis used, awareness, and health-seeking behavior of the study population.

In this study, Trichophyton species was the predominant genus $(79.2 \%)$ among the three genera of dermatophytes followed by genus Epidermophyton species $(20.8 \%)$. It agrees with previous studies performed worldwide, while a study from Spain $^{20}$ reported Trichophyton species as the most prevalent isolate in $76 \%$ of the cases and Microsporum species accounted for the remaing cases.
This variation is mainly due to the difference in the geographical distribution of different dermatophytes.

In this study, males were significantly associated with dermatophytosis infection. Similar findings were reported from previous studies conducted in Harar $^{12}$ and North India. $^{21}$ Male predominance is probably due to higher exposure to infection in schools, public bath, sporting activities, haircuts, sharing of combs, and the type of shoes and socks they use. Our finding is contrary to those reported from Addis Ababa, Ethiopia, ${ }^{13}$ and Nairobi, Kenya ${ }^{6}$, where females had a high prevalence rate of infection than males. The lower prevalence in females in the present study may also be due to the under-reporting of female patients to the hospitals. ${ }^{22}$

In the current study, patients below the age 10 years have higher rates of dermatophytosis infection. It is in agreement with previous reports from Harar, Ethiopia, ${ }^{12}$ Nigeria, ${ }^{23}$ and India. ${ }^{24}$ That may be due to poor hygiene at this age group and the barriers in intake of saturated fatty acids that provide a natural protective mechanism against dermatophyte infection. ${ }^{9}$ In addition, malnutrition is common in this age group, which is a predisposing factor for skin diseases. ${ }^{25}$

In this study, a patients from families having histories of dermatophytosis were the other significant determinant for the development of dermatophytosis. Similar results were obtained from the studies conducted in Addis Zemen Northwest Ethiopia, ${ }^{26}$ Eastern India $^{27}$ and India. ${ }^{9}$ This may be due to frequent close contact between family members facilitating the occurrence of this infection. In addition, the possible sharing of towels, combs, pillows, hats, and head bars may facilitate familial disease transmissions. ${ }^{6}$

Moreover, patients with the history of animal contacts were 1.5 times more likely to develop dermatophytosis infection than those patients who did not have contact. This agrees with studies reported from North India ${ }^{28}$ and Nigeria. $^{23}$ Zoonotic consequences are facilitated by poor compliance with hygiene and health regulations. ${ }^{4}$ But, a study from Rajkot district, India ${ }^{29}$ reported an animal contact was not significantly associated. This variation may be due to the difference iin the study participants in the culture of relations with pet animals. ${ }^{30}$

In this study, patients with hypertension were 2.2 more likely to develop dermatophytosis infection than their counterparts. This finding is in an agreement with a study in Northern parts of Africa. ${ }^{31}$ This may be justified by the side effect of blood pressure medications like amlodipine which causes dryness and itchiness on the skin. ${ }^{32}$ 
Table 4 Bivariate and Multivariable Logistic Regression Analysis of Associated Factors for Dermatophytosis, 2021

\begin{tabular}{|c|c|c|c|c|c|c|c|}
\hline \multirow[t]{2}{*}{ Variables } & \multirow[t]{2}{*}{ Category } & \multicolumn{2}{|c|}{ Dermatophytosis } & \multirow[t]{2}{*}{ COR 95\% Cl } & \multirow[t]{2}{*}{ P-value } & \multirow[t]{2}{*}{ AOR 95\% Cl } & \multirow[t]{2}{*}{ P-value } \\
\hline & & tve (\%) & -ve (\%) & & & & \\
\hline \multirow[t]{2}{*}{ Sex } & Male & $65(33.3)$ & $130(66.7)$ & $2.26(1.28-5.12)$ & 0.11 & $2.5(1.96-4.80)$ & 0.001 \\
\hline & Female & I7 (I8.08) & 77 (81.9) & I & & I & \\
\hline \multirow[t]{4}{*}{ Age } & $0-10$ & 37 (5I.4) & $35(48.6)$ & $4.23(2.106-6.53)$ & 0.000 & $3.5(2.07-5.57)$ & 0.004 \\
\hline & $11-20$ & $23(24.2)$ & $72(75.8)$ & $1.28(1.34-2.76)$ & 0.55 & I.7(0.26-2.209) & 0.62 \\
\hline & $21-30$ & II (16.4) & $56(83.6)$ & $0.78(0.505-3.203)$ & 0.6 & $0.99(0.34-2.88)$ & 0.99 \\
\hline & $>30$ & II (20) & $44(80)$ & I & & I & \\
\hline \multirow[t]{2}{*}{ Residency } & Rural & $37(24.5)$ & II 4 (75.5) & $0.67(0.89-2.49)$ & 0.128 & $2.2(0.22-4.17)$ & 0.069 \\
\hline & Urban & $45(32.6)$ & $93(67.4)$ & I & & I & \\
\hline \multirow[t]{5}{*}{ Occupation } & Farmer & II (I5.5) & 6084.5 & $0.14(0.01-0.89)$ & 0.11 & $2(0.2 I-1.8 I)$ & $0.54 I$ \\
\hline & Student & $50(37.6)$ & $83(62.4)$ & $0.47(0.74-4.08)$ & 0.15 & $0.91(0.64-2.62)$ & 0.192 \\
\hline & House wife & $8(18.2)$ & $36(81.8)$ & $0.17(0.01-2.19)$ & 0.16 & $0.61(0.04-3.05)$ & 0.74 \\
\hline & Other** & $4(16)$ & $21(84)$ & $0.15(0.02-0.93)$ & 0.10 & $0.23(0.74-4.18)$ & 0.41 \\
\hline & Employee & $9(56.25)$ & $74(3.75)$ & I & & I & \\
\hline \multirow[t]{3}{*}{ Education level } & Illitrate & $16(29.09)$ & $39(70.9)$ & $0.96(0.13-2.02)$ & 0.89 & - & - \\
\hline & Primary & $12(22.2)$ & $42(77.8)$ & $0.67(0.03-2.07)$ & 0.46 & - & - \\
\hline & Secondary & $54(30)$ & $126(70)$ & $\mathrm{I}$ & & & \\
\hline \multirow[t]{2}{*}{ Family history of dermatophytosis } & Yes & $72(37.3)$ & $121(62.69)$ & $5.12(2.49-8.72)$ & 0.001 & $2(1.03-5.14)$ & 0.001 \\
\hline & No & $10(10.4)$ & $86(89.6)$ & 1 & & I & \\
\hline \multirow[t]{2}{*}{ Treatment history } & Yes & $27(28.7)$ & $67(7 \mid .3)$ & $0.53(0.04-3.42)$ & 0.015 & $1.9(0.44-2.11)$ & 0.59 \\
\hline & No & $45(43.3)$ & $59(56.7)$ & 1 & & I & \\
\hline \multirow[t]{2}{*}{ Sharing fomites } & Yes & $31(37.8)$ & $51(62.2)$ & $0.49(0.15-3.96)$ & 0.109 & $10.8(0.02-2.46)$ & \multirow[t]{2}{*}{0.72} \\
\hline & No & $15(55.6)$ & $12(44.5)$ & 1 & & I & \\
\hline \multirow[t]{2}{*}{ Animal contact } & Yes & $50(33.1)$ & $10 \mathrm{I}(66.9)$ & $1.62(1.01-3.03)$ & 0.021 & I.5(I.07-4.57) & 0.002 \\
\hline & No & $32(23.4)$ & $105(76.6)$ & I & & I & \\
\hline \multirow[t]{2}{*}{ Diabetic } & Yes & $8(36.4)$ & $14(63.6)$ & I.49(0.27-4.66) & 0.39 & $1.27(0.03-5.22)$ & 0.22 \\
\hline & No & 74 (27.7) & $193(72.3)$ & I & & I & \\
\hline \multirow[t]{2}{*}{ HIV } & Yes & $13(22.4)$ & $45(77.6)$ & $0.68(0.74-2.9)$ & 0.26 & $0.79(0.58-3.86)$ & 0.59 \\
\hline & No & $69(29.87)$ & $162(70.1)$ & 1 & & I & \\
\hline \multirow[t]{2}{*}{ Hypertension } & Yes & $7(58.3)$ & $5(4 I .7)$ & $3.77(1.18-4.86)$ & 0.027 & $2.2(1.12-7.08)$ & 0.03 \\
\hline & No & $75(27.1)$ & 202 (72.9) & I & & I & \\
\hline
\end{tabular}

Note: **Drivers and unemployed.

Abbreviation: $\mathrm{Cl}$, confidence interval. 
On the contrary, a study conducted in India ${ }^{33}$ reported that patients with hypertension did not have a significant relatioship with developing the infection. This was probably due to geographical variation.

In the current study, the prevalence of dermatophytosis among the urban community was higher than in the rural community although no significant association was observed, which is in agreement with a study from southwest Nigeria. ${ }^{34}$ This is maybe because urban people seek medical advice sooner than others due to more awareness and easy accessibility of medical care. While in rural areas, early lesions are neglected and only chronicity forces people to seek medical advice. This finding contradicts a study from India $^{22}$ in which a high rate was observed in the rural community. This variation is maybe due to differences in access to medical care from place to place. ${ }^{25}$

In this study, farmers have a more prevalent rates of dermatophytosis infection than others, although no significant association was observed. However, a study from $\mathrm{Nepal}^{35}$ reported a significantly higher rate of dermatophytosis in farmers. This is probably due to increased physical activities along with sweating, hot weather, and a low degree of hygiene. ${ }^{36}$

This study has a limitation with respect to the laboratory analysis in which we were unable to utilize the most recommended dermatophytes test media. As a result, some fastidious dermatophyte fungal species might have not been isolated from the clinical specimens. Again, not performing antifungal resistance status and molecular identification of the fungi were other limitations of major concern. Moreover, another limitation for this study was also related to the study design and the sampling technique in which temporal associations between the studied variables cannot be assessed and this limits the representativeness of the sample for the general population.

\section{Conclusion}

The present study found more than one in four patients we assessed had dermatophytosis infection. Tinea capitis and Tinea corporis were the most prevalent clinical presentations identified. Trichophyton species was the predominant genus among the three genera of dermatophytes. The infection was considerably high among patients with characterstics involving male sex, below 10 years of age, case of hypertension, family history of dermatophytosis, and contact history with animals. Developing a context appropriate plans and strategies that help to optimally decrease dermatophytosis infection is mandatory with special focus on the high risk populations identified by this study. In these senses, we recommend appropriate measures that help prevent and control the infection such as providing a focused health education to the people at risk and considering conditions that improve the lifestyle of the community including sharing fomites. Moreover, further studies on the prevalence and antifungal resistance status of the infection should be conducted by advanced laboratory techniques that consider molecular identification.

\section{Acknowledgments}

We acknowledge Haramaya University Colleges of Health and Medical Sciences Institutional Health Research Ethical Review Committee for giving the ethical clearance. We also thank study participants and all individuals who have contributed to the successful acomplishment of this study.

\section{Author Contributions}

All authors made a significant contribution to the work reported, whether that is in the conception, study design, execution, acquisition of data, analysis and interpretation, or in all these areas; took part in drafting, revising or critically reviewing the article; gave final approval of the version to be published; have agreed on the journal to which the article has been submitted; and agree to be accountable for all aspects of the work.

\section{Funding}

Data collection of this study was funded by Haramaya University postgraduate directorate.

\section{Disclosure}

The authors report that they have no conflicts of interest.

\section{References}

1. Sumathi T, Shobana C, Mahalakshmi V, et al. Oxidative stress in brains of male rats intoxicated with aluminium and neuromodulating effect of Celastrus paniculatus alcoholic seed extract. Asian J Pharm Clin Res. 2013;6(3):80-90.

2. Burns T, Breathnach S, Cox N, Griffiths C. Rook's Textbook of Dermatology. John Wiley \& Sons; 2008.

3. Havlickova B, Czaika VA, Friedrich MJM. Epidemiological trends in skin mycoses worldwide. J Mycoses. 2008;51:2-15. doi:10.1111/ j.1439-0507.2008.01606.x

4. Kaur I, Thakur K, Sood A, et al. Clinico-mycological profile of clinically diagnosed cases of dermatophytosis in North India. Int J Health Sci Res. 2016;6(8):54-60.

5. Sharma M, Sharma R. Profile of dermatophytic and other fungal infections in Jaipur. Indian $J$ Microbiol. 2012;52(2):270-274. doi:10.1007/s12088-011-0217-z

6. Moto J, Maingi J. Prevalence of Tinea capitis in school going children from Mathare, informal settlement in Nairobi, Kenya. J BMC Res Notes. 2015;8(1):1-4. 
7. Farhan RK, Ahmed T, Mohammad S. Epidemiology of cutaneous candidiasis among patients attending Tikrit Teaching Hospital. Med J Tikrit Univ. 2017;22(1):212-219.

8. Dogo J, Afegbua S, Dung E. Prevalence of Tinea capitis among school children in Nok community of Kaduna State, Nigeria. J Pathog. 2016;2016:1-6. doi:10.1155/2016/9601717

9. Hosthota A, Gowda T, Manikonda R. Clinical profile and risk factors of dermatophytoses. Int $J$ Res Dermatol. 2018;4(4):508-513. doi:10.18203/issn.2455-4529.IntJResDermatol20183860

10. Gesew GT. Prevalence of dermatophytes and non-dermatophyte fungal infection among patients visiting dermatology clinic, at Tikur Anbessa Hospital, Addis Ababa, Ethiopia. Thesis. Addis Ababa University; 2014.

11. Mitiku A. Assessment of disability status of new leprosy patient diagnosed between 2000EFY to 2002EFY from record in Bisidimo Hospital, East Harage. Thesis. University of Gondar; 2014.

12. Alemayehu A, Minwuyelet G, Andualem G. Prevalence and etiologic agents of dermatophytosis among primary school children in Harari Regional State, Ethiopia. J Mycol. 2016;2016:1-5. doi:10.1155/2016/ 1489387

13. Teklebirhan G, Bitew A. Prevalence of dermatophytic infection and the spectrum of dermatophytes in patients attending a tertiary hospital in Addis Ababa, Ethiopia. Int $J$ Microbiol. 2015;2015:1-5. doi: $10.1155 / 2015 / 653419$

14. Araya S, Tesfaye B, Fente D. Epidemiology of dermatophyte and non-dermatophyte fungi infection in Ethiopia. J Clin Cosmet Dermatol. 2020;13:291.

15. Ardakani ME, Ghaderi N, Kafaei P. The diagnostic accuracy of potassium hydroxide test in dermatophytosis. J Basic Med Clin. 2016;5(2):4-6.

16. Shalaby M, El-Din A, El-Hamd M. Isolation, identification, and in vitro antifungal susceptibility testing of dermatophytes from clinical samples at Sohag University Hospital in Egypt. $J$ Electron Physician. 2016;8(6):2557. doi:10.19082/2557

17. Lyngdoh C, Lyngdoh W, Choudhury B, et al. Clinico-mycological profile of dermatophytosis in Meghalaya. Int J Med Public Health. 2013;3(4):254. doi:10.4103/2230-8598.123442

18. Naseri A, Fata A, Najafzadeh M, Shokri H. Surveillance of dermatophytosis in northeast of Iran (Mashhad) and review of published studies. Mycopathologia. 2013;176(3-4):247-253. doi:10.1007/ s11046-013-9688-2

19. Fentaw S, Getachew T, Assefa M. A five-years retrospective study of dermatophytosis and dermatomycoses at the Mycology Referral Laboratory of. EHNRI, Addis Ababa, Ethiopia. J Revista Cenic Ciencias Biológicas. 2010;41:1-7.

20. Costa-Orlandi C, Magalhaes G, Oliveira M, Taylor E, Marques C, de Resende-stoianoff MA. Prevalence of dermatomycosis in a Brazilian tertiary care hospital. J Mycopathologia. 2012;174(5-6):489-497. doi:10.1007/s11046-012-9576-1

21. Lova-Navarro M, Gómez-Moyano E, Pilar LM, et al. Tinea capitis in adults in southern Spain. A 17-year epidemiological study. Revista iberoamericana de micologia. 2016;33(2):110-113.
22. Malik A, Fatima N, Khan P. A clinico-mycological study of superficial mycoses from a tertiary care hospital of a North Indian town. J Virol Mycol. 2014;3:135.

23. Janardhan B, Vani G. Clinico mycological study of dermatophytosis. $J$ Int $J$ Res Med Sci. 2017;5(1):31-39.

24. Oke O, Onayemi O, Olasode O, Omisore A, Oninla OA. The prevalence and pattern of superficial fungal infections among school children in Ile-Ife, South-Western Nigeria. J Dermatol Res Pract. 2014;2014:1-7. doi:10.1155/2014/842917

25. Grover C, Arora P, Manchanda V. Tinea capitis in the pediatric population A study from North India. Indian journal of dermatology. Venereol Leprol. 2010;76(5):527. doi:10.4103/03786323.69078

26. Nawal P, Patel S, Patel M, Soni S, Khandelwal N. A study of superficial mycoses in tertiary care hospital. J Age. 2012;6:11.

27. Saha I, Podder I, Chowdhury SN, Bhattacharya S. Clinico-mycological profile of treatment-naïve, chronic, recurrent and steroid-modified dermatophytosis at a tertiary care centre in Eastern India: an institution-based cross-sectional study. Indian Dermatol Online J. 2021;12(5):714-721. doi:10.4103/idoj.IDOJ 90920

28. Kucheria M, Gupta S, Chhina D, Gupta V, Hans D, Singh K. Clinicomycological profile of dermatophytic infections at a tertiary care hospital in north India. J Int J Com Health and Res. Med. 2016;2 (2): 17-22.

29. Lulekal S, Abera S, Misganaw S, Urgie S. Assessment on prevalence of tinea capitis and its associated factors among school children in Addis Zemen, Northwest Ethiopia. J Cent Afri J Public Health. 2019;5(4):172. doi:10.11648/j.cajph.20190504.16

30. Goyal S, Patel R, Sukhramani P, Kamothi K. Microtubules: a novel target for cancer therapy. J Int J Pharm Sci Res. 2010;1(4):1-21.

31. Nweze E. Dermatophytoses in domesticated animals. J Revista Do Instituto de Medicina Tropical de São Paulo. 2011;53(2):94-99. doi:10.1590/S0036-46652011000200007

32. Nweze E, Eke I. Dermatophytes and dermatophytosis in the eastern and southern parts of Africa. J Med Mycol. 2018;56(1):13-28. doi:10.1093/mmy/myx025

33. Peleli M, Bibli S, Li Z, et al. Cardiovascular phenotype of mice lacking 3-mercaptopyruvate sulfur transferase. $J$ Biochem Pharmacol. 2020;176:113833. doi:10.1016/j.bcp.2020.113833

34. Olutoyin OO, Onayemi O, Gabriel A. Risk factors associated with acquiring superficial fungal infections in school children in South Western Nigeria: a comparative study. J Afri Health Sci. 2017;17 (2):330-336. doi:10.4314/ahs.v17i2.6

35. Mathur M, Kedia S, Ghimire R. A clinico-mycological study of dermatophytic infections in central Nepal. Kathmandu Univ Med J. 2012;10(1):20-23. doi:10.3126/kumj.v10i1.6910

36. Raina D, Gupta P, Khanduri A. A first case of Microsporum ferrugineum causing tinea corporis in Uttarakhand. J Ann Trop Med Health Public. 2016;9(5):351. doi:10.4103/1755-6783.190195
Clinical, Cosmetic and Investigational Dermatology is an international, peer-reviewed, open access, online journal that focuses on the latest clinical and experimental research in all aspects of skin disease and cosmetic interventions. This journal is indexed on CAS.
The manuscript management system is completely online and includes a very quick and fair peer-review system, which is all easy to use. Visit http://www.dovepress.com/testimonials.php to read real quotes from published authors. 